\title{
DETERMINACIÓN DEL EFECTO DE LA CONCENTRACIÓN DE LA CELULASA, CELOBIASA Y DE NaOH EN LA HIDRÓLISIS PARA LA PRODUCCIÓN DE ETANOL A PARTIR DEL RASTROJO DE LA PIÑA
}

\author{
Maureen Córdoba Pérez \\ Manuel E. Molina Córdoba
}

\begin{abstract}
Resumen
El objetivo de esta investigación fue determinar el efecto de la concentración de la celulasa, celobiasa y del hidróxido de sodio en la hidrólisis de la celulosa del rastrojo de piña. El rastrojo de la piña de la variedad MD2 utilizado contiene: $86,4 \%$ de humedad, $12,06 \%$ de cenizas en base seca, $66,2 \%$ de holocelulosa y $8,3 \%$ lignina en base seca libre de extractos. Se realizó el pretratamiento a diferentes concentraciones de $\mathrm{NaOH}$ y diferentes tiempos encontrándose que el rendimiento más alto corresponde a un tiempo de 25 minutos. Para muestras pretratadas con $\mathrm{NaOH}$ al $1,00 \%$ y $1,25 \%(\mathrm{~m} / \mathrm{v})$ a una temperatura de $121^{\circ} \mathrm{C}$ por 25 minutos, concentraciones de celulasa de 10,15 y $20 \mathrm{FPU} / \mathrm{g}$; una relación celulasa:celobiasa (v:v) de 5:1, 7:1 y 9:1 respectivamente, estadísticamente no se encontró diferencia significativa entre la concentración de hidróxido de sodio y la relación de enzima celulasa:celobiasa en el rendimiento de glucosa; por lo que se establece una concentración de $\mathrm{NaOH}$ de 1,00\% en la etapa de pretratamiento y una relación de enzimas de 7:1. En la sacarificación y fermentación simultáneas utilizando cantidades de rastrojo de $(25,10 ; 35,10$ y 50,30$) \mathrm{g} / \mathrm{L}$ se determinó que los rendimientos fueron de $56,26 \% ; 52,45 \%$ y 50,33\% respectivamente; resultados obtenidos al cabo de 25 horas de hidrólisis. La concentración de etanol promedio obtenida es de 1,87\% v/v.
\end{abstract}

Palabras clave: rastrojo de piña; material lignocelulósico; pretratamiento alcalino; etanol celulósico.

\begin{abstract}
The main goal of this research was to determine the effect of cellulase, celobiase and sodium hydroxide concentration in the hydrolysis of cellulose of pineapple's straw. The straw used was obtained from pineapple variety MD2 and had this composition: $86,4 \%$ of water, $12,06 \%$ of ash (d.b), 66,2\% of holocellulose and 8,2\% of lignin (d.b free extracts).A pretreatment was executed at different times and concentrations of $\mathrm{NaOH}$, and it was found that the highest yield is obtained at 25 minutes.For pretreated samples at test conditions of $\mathrm{NaOH}$ at $1,00 \%$ and $1,25 \%(\mathrm{~m} / \mathrm{v})$, $121^{\circ} \mathrm{C}$ for 25 minutes, a cellulase concentration of 10,15 and $20 \mathrm{FPU} / \mathrm{g}$ and cellulase: celobiase ratio (v:v) of 5:1, 7:1 and 9:1, it was found that there is no statistical difference between $\mathrm{NaOH}$ concentration and cellulase: celobiase ratio in the yield for obtaining glucose. It was fixed $\mathrm{NaOH}$ concentration of $1,00 \%$ for the pretreatment stage and enzymes ratio of 7:1.In the simultaneous saccharification and fermentation for different concentrations of straw, it was determined that for 25 hours of hydrolysis, the yields for obtaining glucose were $56,26 \%$ for a straw concentration of $25,10 \mathrm{~g} / \mathrm{L}, 52,45 \%$ for a concentration of $35,10 \mathrm{~g} / \mathrm{L}$ and $50,33 \%$ for a concentration of $50,30 \mathrm{~g} / \mathrm{L}$. The mean ethanol concentration obtained was $1,87 \% \mathrm{v} / \mathrm{v}$.
\end{abstract}

Keywords: pineapple straw; lignocellulosic material; alkaline pretreatment; cellulosic ethanol.

Recibido: 04 de setiembre del 2013 • Aprobado: 13 de febrero del 2014

\section{INTRODUCCIÓN}

La demanda de etanol para su utilización como combustible ha aumentado en los últimos años. Según el Informe del Estado Global de los Renovables (REN21, 2010), la producción de bioetanol en el mundo para el año 2009 sustituyó cerca del 4\% de la producción mundial de gasolinas. La incertidumbre relacionada con la disponibilidad de fuentes de petróleo a largo plazo, la fluctuación en los precios del mismo así como los problemas de contaminación, han promovido el uso de etanol como aditivo de la gasolina. 
Las materias primas para la producción del bioetanol incluyen mieles y jugos ricos en sacarosa de fábricas azucareras, productos agrícolas ricos en almidón y materiales lignocelulósicos. Sin embargo, la producción de etanol a partir de productos que contienen almidón está cuestionada debido a que se está utilizando una fuente de alimentación para la producción de energía, comprometiendo su disponibilidad y aumentando los precios para los consumidores.

Los materiales lignocelulósicos se perfilan como una fuente alternativa que no presenta el problema de los materiales amiláceos y están disponibles en grandes cantidades. Su composición es básicamente lignina, celulosa $\mathrm{y}$ hemicelulosa. La celulosa y hemicelulosa se pueden degradar en azúcares fermentables mediante el uso de enzimas y la aplicación de un pretratamiento previo para exponer las fibras de celulosa a la acción de las enzimas.

Los estudios realizados con diferentes materiales buscan disminuir los costos asociados con el proceso de hidrólisis y fermentación para la producción de etanol. Esto ha llevado a la utilización del método de Sacarificación y Fermentación Simultáneas (SFS), un proceso en el cual hay un aumento en la velocidad de la hidrólisis, la cantidad de enzima requerida es menor y el tiempo de proceso es más corto.

El objetivo de esta investigación es determinar el efecto de la concentración de hidróxido de sodio $(\mathrm{NaOH})$, de celulasa y de la celobiasa en la hidrólisis para la producción de etanol a partir del rastrojo de la piña, utilizando un sistema hidrólisis fermentación.

\section{FUNDAMENTOS TEÓRICOS}

\subsection{Materiales lignocelulósicos}

La lignocelulosa es el componente principal de las paredes celulares vegetales, consiste principalmente en celulosa, hemicelulosa y lignina junto con pequeñas cantidades de pectina, proteínas, clorofila, grasas y cenizas (Kumar, Barret, Delwiche, \& Stroeve, 2009).
El potencial de utilizar diferentes materiales lignocelulósicos han sido reportados por Howard, Abotsi, Jansen \& Howard (2003).

La celulosa es un polisacárido formado por subunidades de D-glucosa unidas por enlaces $\beta-1,4-$ glucosídicos. La unión de las moléculas mediante puentes de hidrógeno en la celulosa forma una estructura lineal ordenada que representa entre un 50-90\% de la celulosa total, la otra parte tiene un arreglo desordenado o amorfo (Foyle, Jennings, \& Mulcahy, 2007). La celulosa constituye entre un 30 a $60 \%$ de la materia seca de la planta (Balat, 2010).

La celobiosa es el dímero repetitivo que constituye las cadenas de celulosa. La celulosa en su forma amorfa es más susceptible a la degradación enzimática (Kumar et al, 2009).

La hemicelulosa es un polímero heterogéneo que representa, en general, $15-35 \%$ de la biomasa de la planta. Consiste en cadenas cortas y ramificadas de azúcares. Contiene azúcares de cinco carbonos, generalmente xilosa y arabinosa y azúcares de seis carbonos (Girío et al, 2010).

La lignina tiene una estructura amorfa que consiste en unidades diferentes de fenilpropano. La función principal de la lignina es dar soporte estructural a la planta, impermeabilidad, resistencia contra ataques microbianos y al estrés oxidativo. Es insoluble en agua y ópticamente inactiva lo que la hace difícil de degradar (Hendricks \& Zeeman, 2009).

\subsection{Rastrojo de piña}

El rastrojo de piña es un material lignocelulósico de desecho del sector agrícola generado en grandes volúmenes. Su degradación es lenta por lo que se ha convertido en un problema ambiental.

El rastrojo corresponde a las hojas de la planta de piña, las cuales son extremadamente fibrosas, tenaces y abrasivas debido a su alto contenido de silicio. Contienen también cordones de fibra, específicos de la piña que le confiere gran resistencia a la torsión (Quesada, Alvarado, Sibaja, \& Vega, 2005).

Alrededor de 1,5 millones de toneladas métricas de rastrojo se transforman en desecho representando más de la mitad de la biomasa 
implicada en el cultivo de la piña (MINAET, MAG, \& PROAGROIN, 2009).

Las prácticas agrícolas aplicadas para eliminar el rastrojo son perjudiciales para el medio ambiente debido a que utilizan herbicidas para destruir la planta y luego se queman.Asu vez estos residuos promueven la proliferación de la mosca de los establos (Stomoxys calcitrans) que afecta la producción de leche y carne al provocar estrés en el ganado.

\subsection{Pretratamiento de materiales lignocelulósicos}

El objetivo del proceso de pretratamiento es romper la estructura de la lignina y la estructura cristalina de la celulosa, de tal forma que los ácidos o enzimas tengan acceso a la celulosa y la hidrolicen (Yu,Vlasenko, Ding, Labavitch \& Shoemaker, 1997; Howard et al., 2003; Mosier et al., 2005).

Hay diferentes tipos de pretratamientodentro de los cuales se encuentra el pretratamiento alcalino. El pretratamiento alcalino tiene la ventaja que se realiza a bajas temperaturas y presiones comparado con otras tecnologías para pretratamiento. Comparado con el pretratamiento ácido, el proceso alcalino causa menos degradación de azúcares y la estructura de la celulosa después del pretratamiento es más densa y termodinámicamente más estable que la celulosa natural (Hendricks \& Zeeman, 2009; Kumar et al, 2009).

\subsection{Hidrólisis y fermentación de materiales lignocelulósicos}

La hidrólisis se puede realizar con el uso de celulasas las cuales son altamente específicas. Los productos son azúcares reductores incluyendo glucosa.

Las celulasas son una mezcla de varias enzimas, los grupos principales son: endogluconasa, exogluconasa o celobiohidrolasa y $\beta$-glucosidasa. Específicamente, los tres tipos de enzimas que incluye una celulasa son: la endo- $\beta$-1,4-glucanasa o 1,4- $\beta$-D-glucanglucanhidrolasa; la exo- $\beta$-1,4glucanasa o 1,4- $\beta$-D-glucancelobiohidrolasa y la $\beta$-1,4-glucosidasa o $\beta$-D-glucósido glucohidrolasa (Ovando \&Waliszewski, 2005).
La celobiosa, uno de los productos de la hidrólisis, inhibe la acción de las glucanasas limitando la degradación de la celulosa, por lo que es necesario utilizar una celobiasa para su hidrólisis (Ovando \& Waliszewski, 2005).

Después de la hidrólisis, los azúcares liberados se fermentan para producir etanol. En el proceso de sacarificación y fermentación simultánea (SSF) los azúcares producidos en la hidrólisis son fermentados inmediatamente por microorganismos, por lo que la formación de los productos inhibidores de la hidrólisis disminuye.

El microorganismo más utilizado para la fermentación es la levadura Saccharomyces cerevisiae. Esta levadura puede fermentar hexosas pero no la xilosa; produce etanol con altos rendimientos (Balat, 2010).

\section{EQUIPO, MATERIALES Y METODOLOGÍA EXPERIMENTAL}

\subsection{Equipo Experimental}

Para está investigación se utilizó como equipos principales:

a) Autoclave: se utiliza para realizar el pretratamiento del material, ésta opera a un ámbito de presión de 0- 3 atm.

b) Incubadora: "Shaker" con control de temperatura $\left(0{ }^{\circ} \mathrm{C}-60{ }^{\circ} \mathrm{C}\right)$ y agitación de $(0-500)$ $\mathrm{rev} / \mathrm{min}$. Este equipo se utiliza en la etapa de hidrólisis.

c) Refractómetro de Abbe: para la determinación de la concentración de etanol mediante el índice de refracción.

\subsection{Materiales}

Para el desarrollo de este trabajo se utilizaron los siguientes materiales:

Rastrojo de piña: El rastrojo se adquirió de una finca productora de piña MD2 ubicada en San Carlos. El material estaba libre de productos químicos. La fibra se extrajo mediante la molienda en una licuadora. 
Enzimas: Para la etapa de hidrólisis se utilizó una celulasa y una celobiasa. La celulasa utilizada es la que se comercializa con el nombre de ACCELLERASE® 1500 y la celobiasa como $\beta$-glucosidasa Novozyme® 188 . La celulasa es producida a partir de una cepa modificada del hongo Trichoderma reesei. Las condiciones óptimas de operación para esta enzima son: temperatura de $50^{\circ} \mathrm{C}$ a $65^{\circ} \mathrm{C}$ y pH entre 4,0 y 5,0 . La cantidad de $\beta$-glucosidasa utilizada se determinó mediante una relación de volumen/ volumen de celulasa: celobiasa.

Levadura: Para la fermentación se utilizó una cepa de Sacharomyces cereviseae, adquirida en un mercado nacional.

\subsection{Métodos de análisis}

Glucosa: La determinación de la concentración de glucosa se realizó utilizando el método de Trinder. Las lecturas se realizan a una longitud de onda de $500 \mathrm{~nm}$, empleando un espectrofotómetro Bio Mate 3.

Azúcares reductores: La determinación de la concentración de azúcares reductores se realizó utilizando el método de Nelson Somogyi (Southgate, 1976). Las lecturas se realizan a una longitud de onda de $500 \mathrm{~nm}$, empleando un espectofotómetro Bio Mate 3.

Pentosas: La determinación de pentosas se realizó mediante el método de Bial modificado por Horecker (Colowick y Kaplan, 1957). La absorbancia se mide a una longitud de onda de $670 \mathrm{~nm}$.

Actividad de la celulasa: Para determinar la actividad de la celulasa se utilizó el método de Adney \& Baker (1996). Con este método, la actividad de la enzima se expresa en unidades FPU (Filter Paper Unit). El sustrato son $50 \mathrm{mg}$ de papel de filtro Whatman $\mathrm{N}^{\circ} 1$. La celulasa se hidroliza durante 60 minutos a un $\mathrm{pH}$ de 4,8 y una temperatura de 50 ${ }^{\circ} \mathrm{C}$. Se debe determinar la concentración de enzima que libere $2 \mathrm{mg}$ de azúcares a las condiciones mencionadas. Los azúcares liberados se cuantifican mediante el método de Nelson Somogyi.

\subsection{Caracterización del rastrojo de la piña}

Para la caracterización de la composición de la piña se utilizan los métodos de la American Testing
Materials (ASTM). Las cenizas se determinan mediante el método ASTM-1102-56, la humedad mediante secado a una temperatura de $105{ }^{\circ} \mathrm{C}$; extractos solubles en agua fría y caliente según el ASTM-1110-84, extractos solubles en etanolciclohexano con el ASTM-1107-56, cantidad de lignina con el ASTM-1104-56 y holocelulosa con el ASTM-1105-96. Las determinaciones se realizaron por triplicado.

\subsection{Metodología Experimental}

\section{Primera etapa:}

Esta etapa tiene como propósito determinar el efecto de diferentes concentraciones de $\mathrm{NaOH}$ en el pretratamiento del material lignocelulósico y escoger aquella que produzca el mayor rendimiento.

Se realiza el pretratamiento del rastrojo con diferentes concentraciones de hidróxido de sodio: $0 \% ; 0,25 \% ; 0,50 \% ; 0,75 \% ; 1,00 \% ; 1,25 \% ; 1,50 \%$ y $1,75 \%$ y $2,00 \%(\mathrm{~m} / \mathrm{v})$. El rastrojo se prepara en cuadros de 1-2 cm de lado, la fibra se extrae y se lava para eliminar todo material adherido, posteriormente se seca. El material seco se pretrata con las diferentes concentraciones de $\mathrm{NaOH}$; el volumen de la solución alcalina es de $10 \mathrm{~mL} / \mathrm{g}$ de rastrojo seco. Estas muestras se introducen en la autoclave por 20 minutos a $121^{\circ} \mathrm{C}$. Luego, se lava el rastrojo con agua hasta que el líquido de lavado sea claro.

Una vez finalizado los lavados, las muestras se secan. La etapa de hidrólisis se realiza en erlenmeyer de $250 \mathrm{~mL}$, que se coloca en la incubadora a $50{ }^{\circ} \mathrm{C}$ con una agitación de $70 \mathrm{rev} / \mathrm{min}$. El pH se mantiene a 4,8 con un buffer de citrato de sodio $0,05 \mathrm{M}$. La cantidad de rastrojo para la hidrólisis es de $11 \mathrm{~g} / \mathrm{L}$. La celulasa se agrega en una concentración de $10 \mathrm{FPU} / \mathrm{g}$ y una relación volumétrica de celulasa: celobiasa de 3:1. El tiempo de hidrólisis fue de 48 horas. Transcurrido este tiempo, se determina la concentración de glucosa mediante el método de Trinder.

\section{Segunda etapa:}

Esta etapa tiene por objetivo definir un tiempo para el pretratamiento del material lig- 
Tabla 1. Composición del rastrojo.

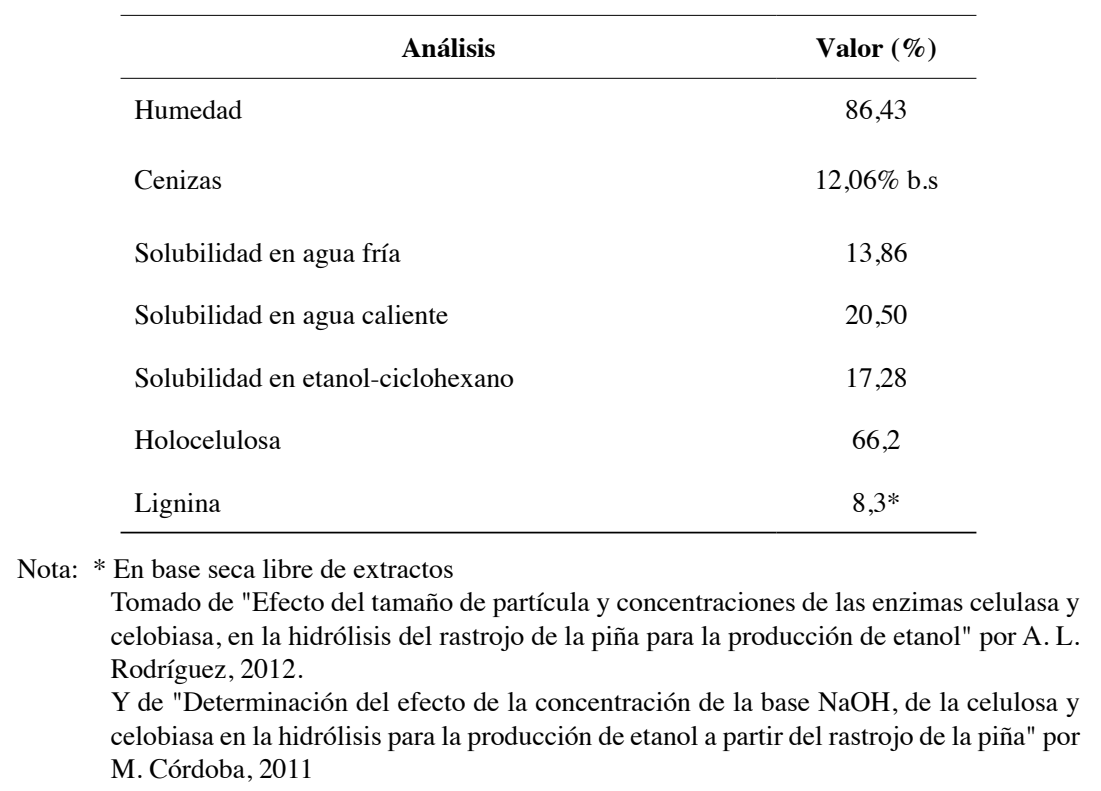

nocelulósico para cada una de las concentraciones de $\mathrm{NaOH}$ utilizadas, a saber $0,75 \% ; 1,0 \%$; $1,25 \%$ y $1,5 \%(\mathrm{~m} / \mathrm{v})$, que produzca el mayor rendimiento; las muestras de rastrojo a cada concentración del álcali se pretratan a 10, 20, 30 y 40 minutos en el autoclave. Se procede de la misma forma que en la etapa anterior para la preparación del material y la hidrólisis.

\section{Tercera etapa:}

El objetivo es evaluar el efecto de la concentración de hidróxido de sodio y la concentración de enzimas en el rendimiento de la hidrólisis del material; se realiza un diseño de bloques por duplicado con dos niveles de concentración de $\mathrm{NaOH}(1,00 \%$ y $1,25 \% \mathrm{~m} / \mathrm{v})$ y tres niveles de concentración de celulasa, que corresponden a una relación volumétrica celulasa: celobiasa de 5:1, 7:1 y 9:1 respectivamente.

\section{Cuarta Etapa:}

El propósito es evaluar la producción de glucosa y etanol utilizando un sistema SSF, utilizando diferentes concentraciones de sustrato.
En esta etapa se realiza la hidrólisis y fermentación simultáneas con las condiciones para el pretratamiento e hidrólisis que producen los mayores rendimientos de glucosa.

El material lignocelulósico se pretrata con una solución de hidróxido de sodio al $1,00 \%$ por 25 minutos y $121{ }^{\circ} \mathrm{C}$; la hidrólisis se realiza con una concentración de celulasa de $15 \mathrm{FPU} / \mathrm{g}$ y una relación volumétrica celulasa: celobiasa de 7:1. La temperatura de reacción es de $50^{\circ} \mathrm{C}$ en la etapa de hidrólisis y $32^{\circ} \mathrm{C}$ en la fermentación a un pH de 4,8. La agitación se mantiene en $70 \mathrm{rev} / \mathrm{min}$. El tiempo de hidrólisis fue de 25 horas y el de fermentación de 30 horas. Las cantidades de sustrato utilizadas fueron: $25,10 \mathrm{~g} / \mathrm{L}, 35,10 \mathrm{~g} / \mathrm{L}$ y $50,30 \mathrm{~g} / \mathrm{L}$.

\section{ANÁLISIS DE RESULTADOS}

\subsection{Composición del rastrojo.}

En la Tabla 1 se presentan algunos de los parámetros evaluados. Sun \& Cheng (2002) reportan valores para la lignina en el olote de maíz de 15\%; para desechos de pastos, 18 a 30\%; paja de trigo, $15 \%$ y para pastos de crecimiento rápido, $12 \%$. Tomando estos valores como referencia se 


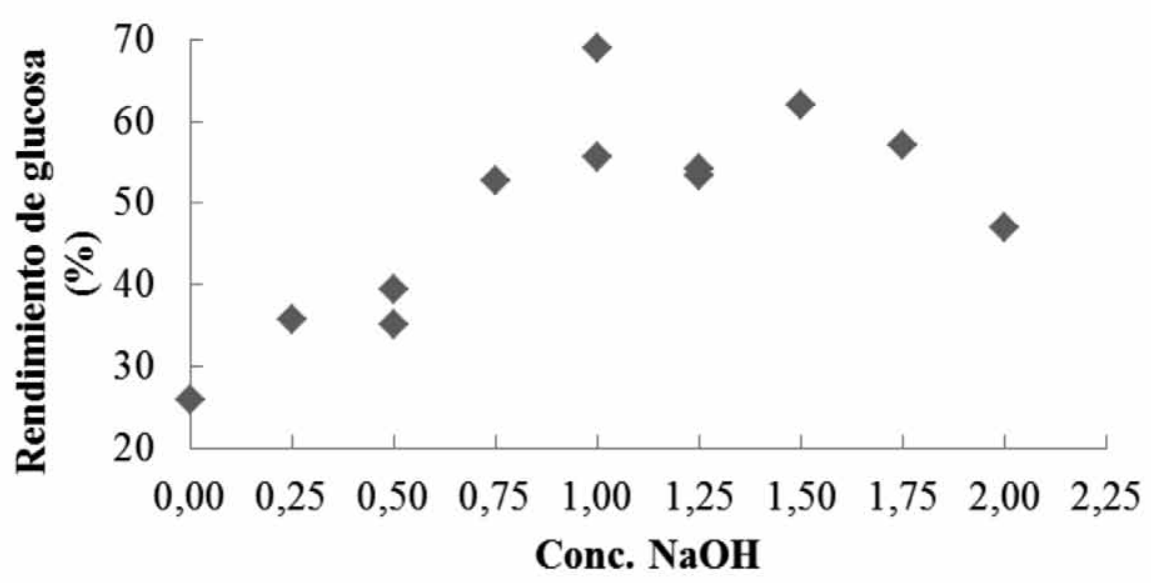

Figura 1. Rendimientos de glucosa a diferentes concentraciones de hidróxido de sodio.

encuentra que el contenido de lignina en el rastrojo es menor, aspecto que favorece el proceso de deslinificación durante el pretratamiento. Mientras que para la holocelulosa, reportan valores de $80 \%$ para el olote de maíz; $80 \%$ para la paja de trigo y $76 \%$ para pastos de crecimiento rápido un $76 \%$; todos estos valores son mayores al valor de $66,2 \%$ para el rastrojo de piña.

\subsection{Pretratamiento a diferentes concentracio- nes de $\mathrm{NaOH}$}

Con el objetivo de determinar los niveles de concentración de hidróxido de sodio a utilizar en la etapa de pretratamiento, se realizan ensayos a diferentes concentraciones de $\mathrm{NaOH}$, manteniendo fijo el tiempo de pretratamiento y las condiciones del proceso de hidrólisis.

La Figura 1 muestra los rendimientos de glucosa después de 48 horas de hidrólisis enzimática, con respecto a la concentración de hidróxido de sodio utilizada en el pretratamiento. Se observa que hay un aumento en el rendimiento de glucosa hasta una concentración de 1,00\% de $\mathrm{NaOH}$, posteriormente para concentraciones mayores de $\mathrm{NaOH}$ en el pretratamiento hay una disminución de este rendimiento.

El rendimiento más alto es de $62,28 \%$ para una concentración de $1,00 \%$ de hidróxido de sodio y el más bajo corresponde, como es de esperar, a la concentración de hidróxido de sodio de $0 \%$ donde el rendimiento es de $27,64 \%$. El rendimiento para las concentraciones de $1,25 \%$ y $1,50 \%$ son de $53,80 \%$ y $61,93 \%$ respectivamente, este último resultado es cercano al obtenido con la concentración de $\mathrm{NaOH}$ de $1,00 \%$.

La disminución en el rendimiento de glucosa al aumentar la concentración de hidróxido de sodio concuerda con lo reportado por Nlewem \& Thrash (2010), Amador (2009) y Torres (2009), quienes determinaron que un aumento en la concentración de hidróxido de sodio provoca una degradación irreversible de la celulosa que se pierde en los lavados del material por lo que los rendimientos de la hidrólisis disminuyen.

El contenido de lignina en el rastrojo de la piña es de $8,3 \%$, un porcentaje bajo si se compara con las especies maderables, por lo que no es necesario utilizar altas concentraciones de $\mathrm{NaOH}$ en el pretratamiento como lo menciona Sun \& Cheng (2002). Los autores indican que los pretratamientos con hidróxido de sodio diluido originan buenos rendimientos de glucosa para materiales con bajo contenido de lignina (10-18\%).

En la Figura 2 se muestra micrografías de rastrojo sin pretratar, se observa tejido epidérmico y abundante tejido parenquimático, el cual es parte de la anatomía de las especies no maderables como el rastrojo de la piña. Durante el proceso de trituración para la extracción de la fibra, se liberan algunos haces de fibras. 

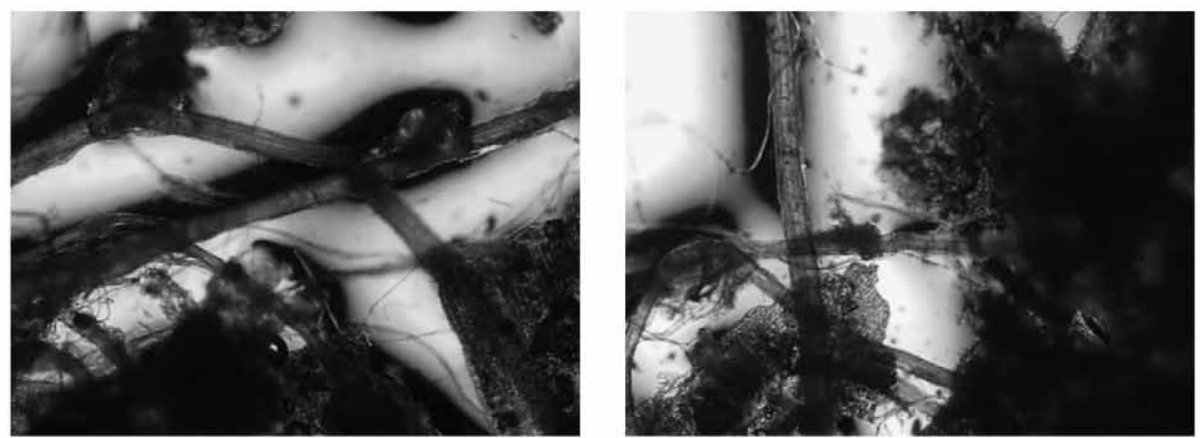

Figura 2. Micrografía de la muestra de rastrojo sin pretratamiento (aumento 4X).

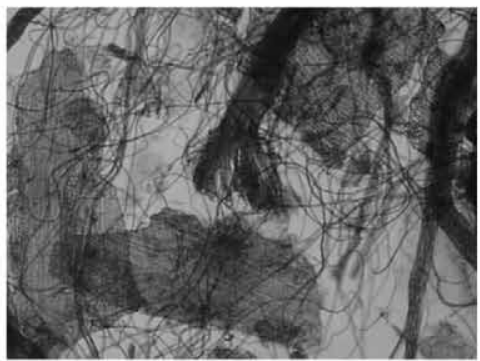

a)

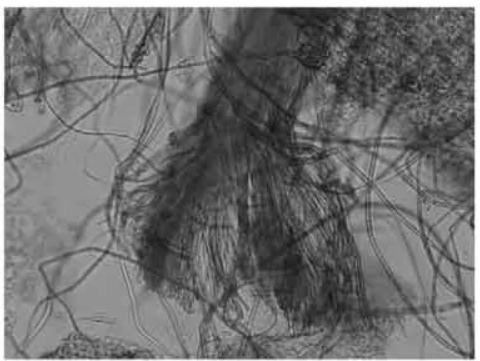

b)

Figura 3. Micrografía de la muestra de rastrojo pretratada con hidróxido de sodio al $1,00 \%(\mathrm{~m} / \mathrm{v})$, a) aumento $4 \mathrm{X}, \mathrm{b})$ aumento $10 \mathrm{X}$.

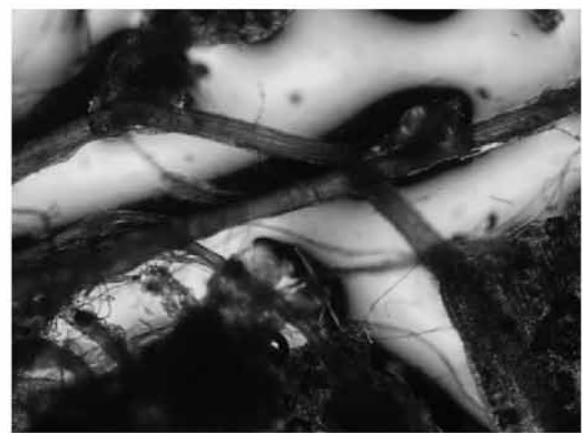

a)

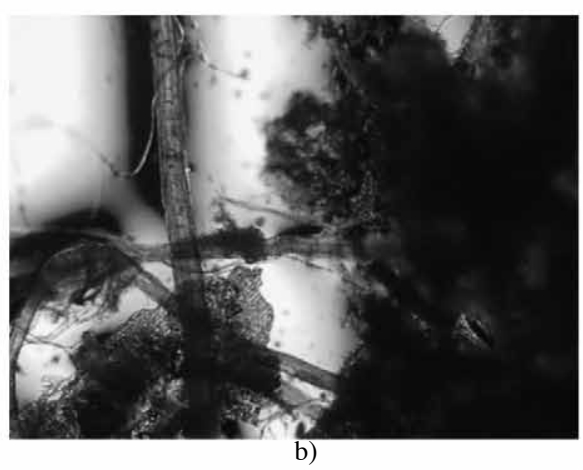

b)

Figura 4. Micrografía de la muestra de rastrojo pretratada con hidróxido de sodio al $1,25 \%(\mathrm{~m} / \mathrm{v})$, aumento $4 \mathrm{X}$. 

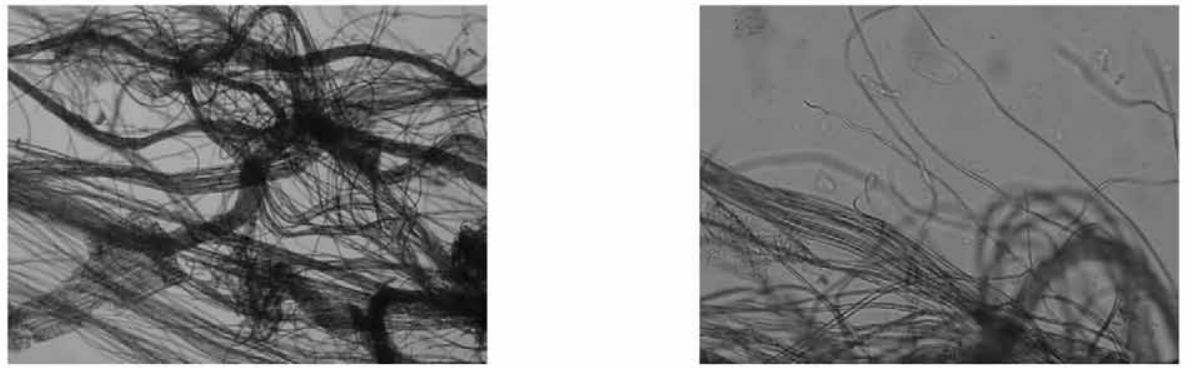

Figura 5. Micrografía de la muestra de rastrojo pretratada con hidróxido de sodio al $1,50 \%(\mathrm{~m} / \mathrm{v})$, a) aumento $4 \mathrm{X}, \mathrm{b})$ aumento $10 \mathrm{X}$.
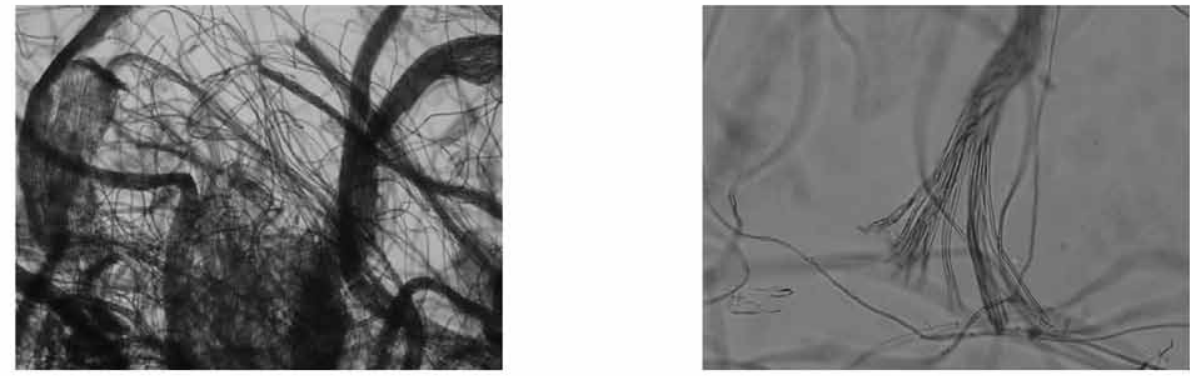

Figura 6. Micrografía de la muestra de rastrojo pretratada con hidróxido de sodio al $2,00 \%(\mathrm{~m} / \mathrm{v})$, a) aumento $4 \mathrm{X}, \mathrm{b})$ aumento $10 \mathrm{X}$.

En las Figuras 3, 4, 5 y 6, se presentan las micrografías de las muestras de rastrojo pretratadas. Se observa que hay tejido parenquimático junto con los haces de fibras. Éstas corresponden a muestras pretratadas con $1,00 \%, 1,25 \%, 1,50 \%$ y $2,00 \%$ de $\mathrm{NaOH}$.

Según lo observado en las micrografías, utilizar concentraciones de $1,50 \%$ y $2,00 \%$ origina que los haces de fibras estén más sueltos con respecto a las otras concentraciones.

De acuerdo a los resultados obtenidos en esta parte de la investigación se toma como niveles para la tercera etapa experimental, concentraciones de $\mathrm{NaOH}$ de $1,00 \%$ y $1,25 \%$ en el pretratamiento del material.

\subsection{Determinación de tiempos de pretratamiento}

En esta segunda etapa, el propósito es establecer un tiempo para el pretratamiento; se realizaron corridas a diferentes tiempos y concentraciones de $\mathrm{NaOH}$. En la Figura 7 se muestra la concentración de glucosa y el tiempo de pretratamiento para cada concentración.

En el caso de las concentraciones de $\mathrm{NaOH}$ de $0,75 \%$ y $1,00 \%$, el máximo rendimiento de glucosa es de $67,8 \%$ y $70,84 \%$ respectivamente para un tiempo de 30 minutos de pretratamiento.

Para la concentración de $1,25 \%$ de $\mathrm{NaOH}$, la máxima concentración de glucosa se alcanza a los 


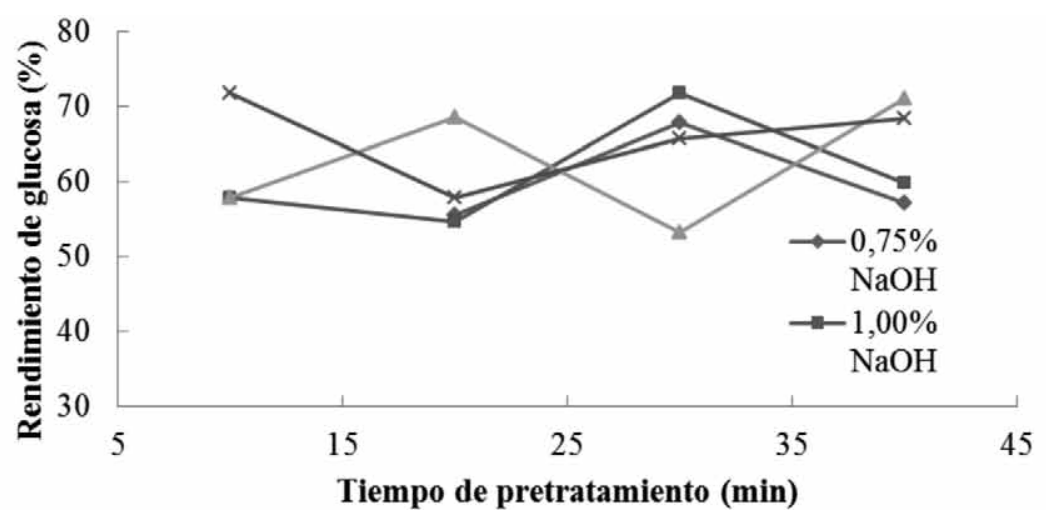

Figura 7. Rendimientos de glucosa a diferentes tiempos y concentraciones de $\mathrm{NaOH}$ en la etapa de pretratamiento.

Tabla 2. Resultados del análisis de Varianza.

\begin{tabular}{lccccc}
\hline \multicolumn{1}{c}{$\begin{array}{c}\text { Origen de las } \\
\text { variaciones }\end{array}$} & $\begin{array}{c}\text { Suma de } \\
\text { cuadros }\end{array}$ & $\begin{array}{c}\text { Grados de } \\
\text { libertad }\end{array}$ & $\begin{array}{c}\text { Promedio de los } \\
\text { cuadrados }\end{array}$ & FCalc & F0,05 \\
\hline $\begin{array}{l}\text { Tiempo de } \\
\text { pretratamiento (min) }\end{array}$ & 1,60 & 2 & 0,801 & 1,00 & 5,14 \\
$\begin{array}{l}\text { Concentración de } \\
\text { NaOH }(\% \mathrm{~m} / \mathrm{v})\end{array}$ & 0,38 & 3 & 0,125 & 0,16 & 4,76 \\
Error & 4,28 & 6 & 0,797 & & \\
Total & 6,76 & 11 & & & \\
\hline
\end{tabular}

40 minutos de pretratamiento que corresponde a un rendimiento promedio de $71,03 \%$; el rendimiento de glucosa para esta concentración para un tiempo de pretratamiento de 20 minutos es de 2,52 unidades menor. Se puede señalar que la reducción en rendimiento porcentual es pequeño, considerando que el tiempo de pretratamiento se reduce a la mitad.

Para la concentración de $\mathrm{NaOH}$ de $1,50 \%$ el mayor rendimiento de glucosa se obtiene a los 10 minutos de pretratamiento, produciéndose una reducción del mismo al aumentar el tiempo.

Con estos resultados, se encuentra que el tiempo de pretratamiento está entre 20 y 30 minutos, ya que a estos tiempos es donde se obtiene la mayor concentración de glucosa y por ende los mayores rendimientos.
Los tiempos de pretratamiento en estudios realizados con bagazo de caña son mayores a los obtenidos en la presente investigación; los rendimientos alcanzados son similares a los de Torres (2009) y Amador (2009). Esto tendría eventualmente un impacto positivo en el aspecto económico.

Se realizó un análisis de varianza con los rendimientos de glucosa obtenidos en esta etapa, encontrándose que no hay efectos significativos entre la concentración de $\mathrm{NaOH}$ y el tiempo de pretratamiento, por lo que se decide utilizar para las siguientes etapas un tiempo de pretratamiento de 25 minutos. El análisis de varianza (ANOVA) se presenta en la Tabla 2. 


\subsection{Determinación del efecto de la concentración de $\mathrm{NaOH}$ y de celulasa y celobiasa en la hidrólisis enzimática del rastrojo de la piña}

Una tercera etapa experimental se estableció para determinar el efecto de la concentración de $\mathrm{NaOH}$ en el pretratamiento del rastrojo de la piña y de la concentración de celulasa y celobiasa en la hidrólisis enzimática para la producción de etanol; se realizó esta etapa mediante un diseño de bloques.

Uno de los problemas de la hidrólisis enzimática es la inhibición de la actividad de la celulasa debido a la presencia de la celobiosa que impide la acción de las glucanasas limitando la degradación de la celulosa. Es por esto que se utiliza en conjunto con la celulasa, la celobiasa con el propósito que hidrolice la celobiosa a glucosa conforme se va liberando en el proceso de hidrólisis.

Los niveles de concentración de $\mathrm{NaOH}$ utilizados son $1,00 \%$ y $1,25 \%$, así como la concentración de celulasa de $10 \mathrm{FPU} / \mathrm{g}, 15$ FPU/g y $20 \mathrm{FPU} / \mathrm{g}$ que al mantener el volumen de celobiasa fijo $(50 \mu \mathrm{L} / \mathrm{g})$, resulta en una relación celulasa: celobiasa de 5:1, 7:1 y 9:1 v:v respectivamente.

En la Tabla 3 se muestran los rendimientos de glucosa. Del análisis estadístico, se obtiene que ni la relación celulasa: celobiasa, ni la concentración de $\mathrm{NaOH}$ en el pretratamiento

Tabla 3. Rendimientos de glucosa.

\begin{tabular}{c|cc}
\hline \multicolumn{2}{c}{ Relación celulasa: celobiasa $(\mathbf{v}: \mathbf{v})$} & \multicolumn{2}{c}{ Concentración de $\mathbf{N a O H}$} \\
\multicolumn{1}{c}{$\mathbf{1 , 0 0 \%}$} & $\mathbf{1 , 2 5 \%}$ \\
\hline $5: 1$ & 54,47 & 54,74 \\
$7: 1$ & 60,17 & 56,55 \\
$7: 1$ & 54,74 & 60,71 \\
$9: 1$ & 58,63 & 60,71 \\
$9: 1$ & 50,31 & 54,47 \\
\hline
\end{tabular}

Tabla 4. Análisis de Varianza.

\begin{tabular}{lccccc}
\hline & $\begin{array}{c}\text { Suma de } \\
\text { cuadros }\end{array}$ & $\begin{array}{c}\text { Grados de } \\
\text { libertad }\end{array}$ & $\begin{array}{c}\text { Promedio de los } \\
\text { cuadrados }\end{array}$ & Fcalc & F0,05 \\
\hline Relación celulasa: celobiasa & 54,75 & 2 & 27,37 & 4,22 & 5,14 \\
Concentración de NaOH & 23,05 & 1 & 23,05 & 3,56 & 5,99 \\
Interacción & 37,46 & 2 & 18,73 & 2,89 & 5,14 \\
Dentro del grupo & 38,90 & 6 & 6,48 & & \\
& & & & & \\
Total & 154,15 & 11 & & & \\
\hline
\end{tabular}


son significativas. La interacción entre las dos variables tampoco es significativa. Los resultados del análisis de varianza se presentan en la Tabla 4.

El rendimiento promedio para la concentración de $\mathrm{NaOH}$ al $1,00 \%$ es de $54,82 \%$ y para la concentración de $1,25 \%$ es de $57,59 \%$. Por otra parte, el rendimiento para la relación volumétrica celulasa:celobiasa de 5:1 correspondiente a una concentración de celulasa de 10 FPU/g es de 56,55\% en promedio, para la concentración de $15 \mathrm{FPU} / \mathrm{g}$ es de $58,63 \%$ (relación de enzimas $7: 1$ ) y para la concentración de 20 FPU/g es de 53,43\% (relación de enzimas 9:1).

El rendimiento de glucosa para la concentración de celulasa de $20 \mathrm{FPU} / \mathrm{g}$ es menor que los rendimientos de glucosa para las concentraciones de celulasa de $15 \mathrm{FPU} / \mathrm{g}$ y 10 FPU/g. Illanes (1994) citado por Torres (2009) menciona que entre mayor es la concentración de enzima, el proceso de hidrólisis es más rápido, sin embargo, según Tolan y Foody (1999), hay un límite superior en cuanto a la conversión de materiales lignocelulósicos. En este punto, la accesibilidad de la enzima a la celulosa es limitada, por lo que aunque se agregue un volumen mayor de enzima, los rendimientos no aumentan.

Al no ser significativa la concentración de hidróxido de sodio en el rendimiento de glucosa en el proceso de hidrólisis, se trabajará en los siguientes ensayos con una concentración de $1,00 \%$. En cuanto a la relación celulasa:celobiasa se utilizará un nivel intermedio, en este caso 15 FPU/g que corresponde a una relación de 7:1.

Tropper (2010), utilizando bagazo de caña obtuvo un rendimiento máximo de $53,98 \%$ de glucosa al utilizar una relación de enzimas de 5:1 después de 40 horas de hidrólisis y una concentración de sustrato de $45 \mathrm{~g} / \mathrm{L}$.

\subsection{Sacarificación y fermentación simultáneas}

\section{Concentración de glucosa y pentosas al inicio de} la fermentación

Las condiciones utilizadas para la fermentación y la consecuente producción de etanol son: para el pretratamiento una concentración de hidróxido de sodio de $1,00 \%$ $(\mathrm{m} / \mathrm{v})$, en la etapa de hidrólisis una concentración de celulasa de $15 \mathrm{FPU} / \mathrm{g}$ de rastrojo seco pretratado y una relación celulasa:celobiasa de 7:1 (v:v).

$\mathrm{Al}$ inicio de la fermentación, las concentraciones y rendimientos de glucosa se presentan en la Tabla 5.

Los rendimientos de glucosa son menores para una cantidad de rastrojo de 50,30 g/L. En la Figura 8 se observa que hay una tendencia lineal al aumento

Tabla 5. Concentración y rendimiento de glucosa al inicio de la fermentación.

\begin{tabular}{ccccc}
\hline $\begin{array}{c}\text { Cantidad de Rastrojo } \\
(\mathbf{g} / \mathbf{L})\end{array}$ & $\begin{array}{c}\text { Conc. de } \\
\text { glucosa } \\
(\mathbf{g} / \mathbf{L})\end{array}$ & Rendimiento(\%) & Varianza & $\begin{array}{c}\text { Desviación } \\
\text { estándar }\end{array}$ \\
\hline 25,10 & 13,91 & 55,43 & 1,38 & 1,17 \\
25,10 & 14,33 & 57,09 & & 4,46 \\
35,10 & 19,56 & 55,73 & 21,52 & \\
35,10 & 17,26 & 49,17 & & 0 \\
50,30 & 25,31 & 50,33 & 0 & \\
50,30 & 25,31 & 50,33 & & \\
\hline
\end{tabular}




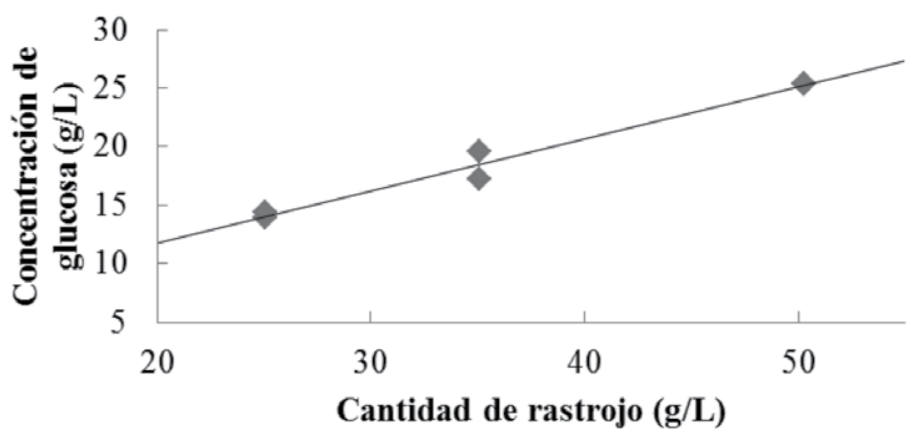

Figura 8. Concentración de glucosa en el tiempo cero de fermentación.

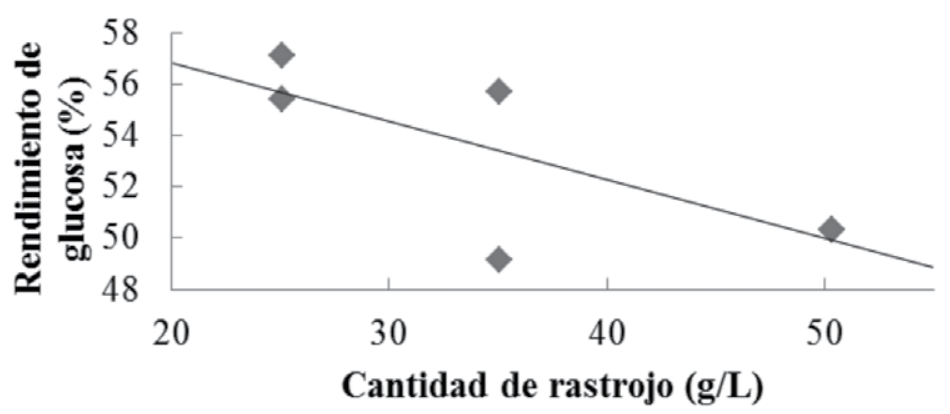

Figura 9. Rendimientos de glucosa en el tiempo cero de fermentación.

de la concentración de glucosa con el aumento de la cantidad de rastrojo. Sin embargo, al considerar el rendimiento de glucosa contra la cantidad de rastrojo, la tendencia es decreciente como se muestra en la Figura 9.

Esto sucede porque el aumento en la carga de rastrojo no implica un aumento de la misma magnitud en la concentración de glucosa, por lo que los rendimientos disminuyen al aumentar la concentración del sustrato, como consecuencia del aumento de la cantidad de material parenquimatoso y otras sustancias que no contribuyen en el proceso de hidrólisis.
Para las cantidades de rastrojo de 25,10 $\mathrm{g} / \mathrm{L}, 35,10 \mathrm{~g} / \mathrm{L}$ y $50,30 \mathrm{~g} / \mathrm{L}$, las concentraciones promedio de glucosa son de $14,12 \mathrm{~g} / \mathrm{L}, 18,41 \mathrm{~g} / \mathrm{L}$ y de $25,31 \mathrm{~g} / \mathrm{L}$ respectivamente.

Si se aplica un ajuste lineal a los datos de la Figura 9, se obtiene el siguiente modelo con un coeficiente de correlación de 0,9016 :

$$
R_{G}=-0,2412 \cdot C n_{R}+62,751
$$

Donde $R_{G}$ se refiere al rendimiento de glucosa y $\mathrm{Cn}_{\mathrm{R}}$ a la carga o cantidad de rastrojo. 


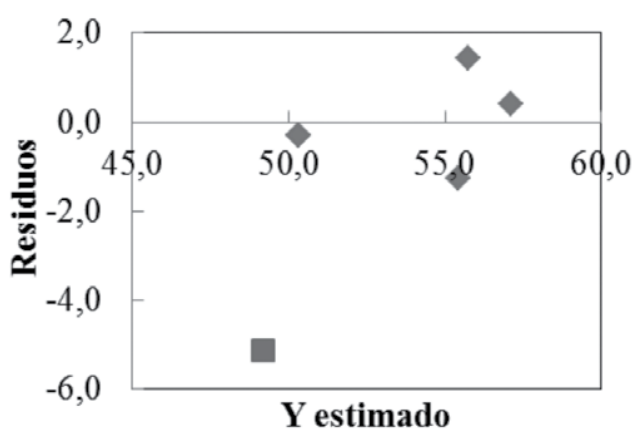

a) Gráfico de residuos

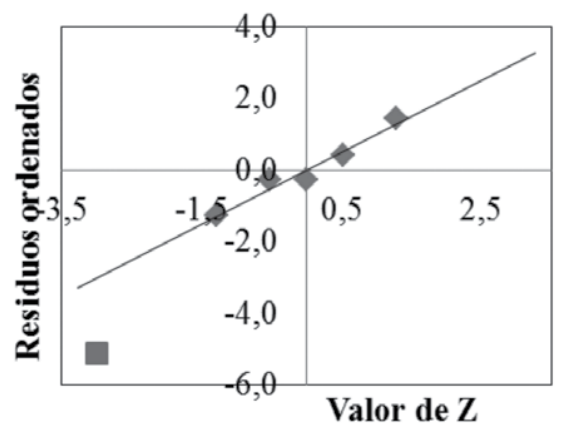

b) Gráfico de normalidad

Figura 10. Análisis de residuos para el rendimiento de glucosa.

Tabla 6. Concentración y rendimiento de pentosas al inicio de la fermentación.

\begin{tabular}{ccc}
\hline $\begin{array}{c}\text { Cantidad de rastrojo } \\
(\mathbf{g} / \mathbf{L})\end{array}$ & Conc. de pentosas $(\mathbf{g} / \mathbf{L})$ & $\begin{array}{c}\text { Rendimiento de pentosas } \\
(\mathbf{\%})\end{array}$ \\
\hline 25,10 & 12,69 & 50,55 \\
25,10 & 11,86 & 47,25 \\
35,10 & 10,73 & 30,58 \\
35,10 & 10,46 & 29,80 \\
50,30 & 13,19 & 26,22 \\
50,30 & 12,56 & 25,16 \\
\hline
\end{tabular}

Para este modelo, no se toma en cuenta el rendimiento de $49,17 \%$ ya que se desvía del modelo. Con esta relación se calculan los rendimientos para cada una de las cantidades de rastrojo en estudio y luego se realiza el análisis de los residuos. La Figura 10 muestra el gráfico de residuos y el gráfico de normalidad.

Se determinó la concentración de pentosas al inicio de la fermentación. Los resultados se muestran en el Tabla 6.

Las concentraciones de pentosas son similares para cada una de las cargas de rastrojo. La concentración promedio de pentosas es de 12,27 g/L,
$10,60 \mathrm{~g} / \mathrm{L}$ y $12,92 \mathrm{~g} / \mathrm{L}$ para las cargas de rastrojo de $25,10 \mathrm{~g} / \mathrm{L}, 35,10 \mathrm{~g} / \mathrm{L}$ y de $50,30 \mathrm{~g} / \mathrm{L}$ respectivamente. Debido a que los rendimientos se calculan con respecto a la cantidad inicial de rastrojo, estos son mayores para la menor carga $(25,10 \mathrm{~g} / \mathrm{L})$. En la Figura 11 se muestra este comportamiento.

En la Figura 12 se presentan micrografías, en ellas se observa tejido parenquimático junto a los haces de fibras que aporta pentosas.

Una opción para la reducción de material parenquimático es someter previamente el rastrojo a una molienda y mejorando las condiciones de lavado se contribuye a que las 


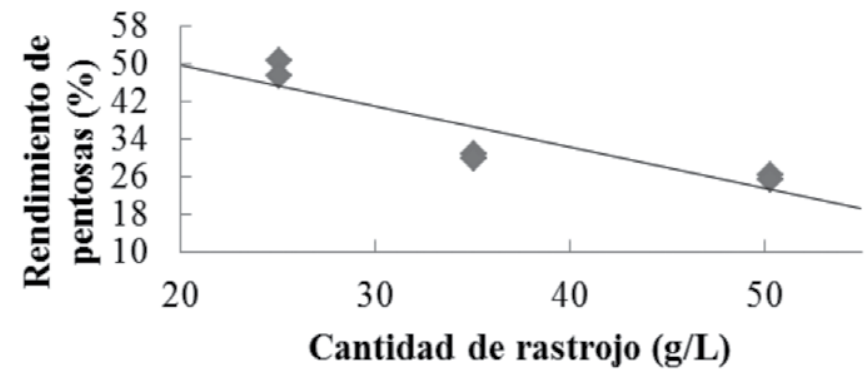

Figura 11. Rendimientos de pentosas al inicio de la fermentación.

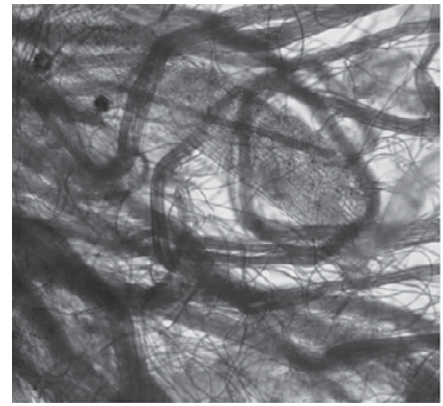

a) Aumento $4 X$

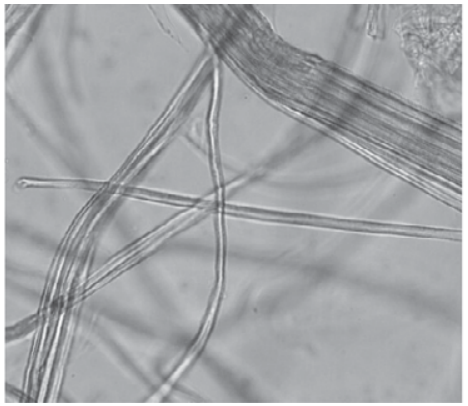

b) Aumento $10 \mathrm{X}$

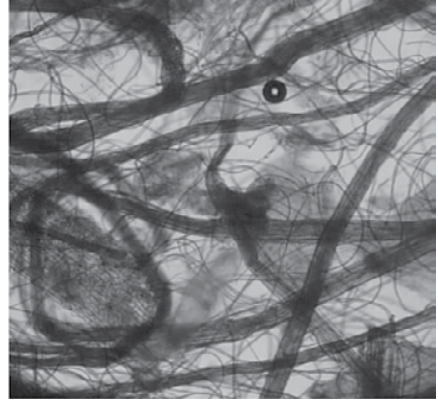

c) Aumento 4X

Figura 12. Micrografías de la fibra petratada con $1,00 \%(\mathrm{~m} / \mathrm{v})$ de $\mathrm{NaOH}$.

Tabla 7. Concentración de etanol para las diferentes cantidades de rastrojo.

\begin{tabular}{cc}
\hline Cantidad de rastrojo $(\mathbf{g} / \mathbf{L})$ & Cantidad de rastrojo $(\mathbf{g} / \mathbf{L})$ \\
\hline 25,10 & 2,00 \\
25,10 & 1,80 \\
35,10 & 1,80 \\
35,10 & 1,80 \\
50,30 & 2,00 \\
50,30 & 1,80 \\
\hline
\end{tabular}


Tabla 8 Concentración de glucosa y azúcares reductores al finalizar la fermentación.

\begin{tabular}{ccc}
\hline $\begin{array}{c}\text { Cantidad de rastrojo } \\
(\mathbf{g} / \mathbf{L})\end{array}$ & Conc. de glucosa $(\mathbf{g} / \mathbf{L})$ & $\begin{array}{c}\text { Conc. de azúcares reductores } \\
(\mathbf{g} / \mathbf{L})\end{array}$ \\
\hline 25,10 & 0,36 & 1,42 \\
25,10 & 0,36 & 1,44 \\
35,10 & 0,39 & 1,12 \\
35,10 & 0,44 & 1,25 \\
50,30 & 0,59 & 1,17 \\
50,30 & 4,34 & 8,63 \\
\hline
\end{tabular}

fibras estén más limpias en cuanto al tejido parenquimático, para ello puede utilizarse una pila holandesa para trabajo a nivel de laboratorio. Estas acciones contribuyen a mejorar la disponibilidad de la celulosa para su hidrólisis.

\subsection{Producción de etanol}

El tiempo de hidrólisis fue de 25 horas, después de este tiempo se procedió a agregar los nutrientes y la levadura para dar inicio a la etapa de fermentación. En la Tabla 7 se muestra la concentración de etanol.

Se obtiene una concentración promedio de etanol de $1,87 \%(\mathrm{v} / \mathrm{v})$, que es una concentración baja si se compara con resultados obtenidos del orden de $7 \%$ a $8 \%$ con otras fuentes de carbohidratos utilizadas en la industria. Para favorecer los rendimientos en la producción de etanol, podría utilizarse levaduras como Pichia stipitis que fermenta la xilosa logrando obtener rendimientos cercanos al teórico de 0,51 g etanol/g xilosa (Chu \& Lee, 2007).

Las concentraciones promedio de glucosa al finalizar el proceso de fermentación son $0,36 \mathrm{~g} / \mathrm{L}, 0,42 \mathrm{~g} / \mathrm{L}$ y $2,47 \mathrm{~g} / \mathrm{L}$ para las cargas de rastrojo de 25,10 g/L, 35,10 g/L y 50,30 g/L respectivamente; para esas mismas cargas las concentraciones promedio de azúcares reductores son de 1,43 g/L, 1,19 g/L y 4,90 $\mathrm{g} / \mathrm{L}$ respectivamente. Los resultados muestran que prácticamente toda la glucosa se consumió en la etapa de fermentación. En la Tabla 8 se presentan estos resultados.

\section{CONCLUSIONES}

- Al pretratar el rastrojo de la piña por un tiempo de 20,30 y 40 minutos con concentraciones de $\mathrm{NaOH}$ de $0,75 \%, 1,00 \%$, $1,25 \%$ y $1,50 \%(\mathrm{~m} / \mathrm{v})$ a una temperatura de $120{ }^{\circ} \mathrm{C}$ y $1,5 \mathrm{~atm}$, estadísticamente, ni el tiempo ni la concentración de $\mathrm{NaOH}$ son significativos respecto a los rendimientos de glucosa. Los mayores rendimientos de glucosa se alcanzan a 20 y 30 minutos de pretratamiento.

- No hay diferencia estadística entre utilizar una concentración de $1,00 \%$ y $1,25 \%$ de hidróxido de sodio en el pretratamiento de las fibras del rastrojo de piña.

- Estadísticamente, no hay diferencia entre utilizar una relación celulasa: celobiasa (v:v) de 5:1, 7:1 y 9:1 en la hidrólisis del rastrojo de la piña.

- $\quad \mathrm{Al}$ aumentar la cantidad de rastrojo hay un aumento en la concentración de glucosa, asî para cantidades de rastrojo de $25,10 \mathrm{~g} / \mathrm{L}$, $35,10 \mathrm{~g} / \mathrm{L}$ y $50,30 \mathrm{~g} / \mathrm{L}$ la concentración de glucosa promedio es de $14,12 \mathrm{~g} / \mathrm{L}, 18,41 \mathrm{~g} / \mathrm{L}$ y de $25,31 \mathrm{~g} / \mathrm{L}$, utilizando una concentración de celulasa de $15 \mathrm{FPU} / \mathrm{g}$ y una relación celulasa:celobiasa de 7:1 (v:v). 
- Puesto que el rastrojo de piña contiene material parenquimático que interfiere tanto en el pretratamiento como en la hidrólisis es necesario mejorar los sistemas de agitación y de lavado.

- Debido a los bajos resultados en la producción de etanol, sería conveniente someter el material a procesos de molienda que contribuyan a la eliminación del material parenquimatoso y que aumente el área superficial para una mejor exposición a las enzimas.

- Al realizar la sacarificación y fermentación simultáneas a diferentes concentraciones de rastrojo se obtuvo una concentración promedio de etanol de $1,87 \% \mathrm{v} / \mathrm{v}$.

- Aunque se alcanzaron altos rendimientos de glucosa, las concentraciones de etanol son bajas comparadas con las concentraciones que se obtienen con otros sustratos utilizados en la industria, razón por la que es importante utilizar otra levadura que fermente xilosas.

\section{BIBLIOGRAFÍA}

Amador, J.L. (2009). Evaluación del efecto del tamaño de partícula, tiempo de pretratamiento y concentración de hidróxido de sodio en la hidrólisis enzimática del bagazo de caña de azúcar.

Adney, B. \& Baker, J. (1996).Measurement of Cellulase Activities. Colorado: National Renewable Energy Laboratory.

Balat, M. (2010). Production of bioethanol from lignocellulosic materials via the biochemical pathway: A review (Versión electrónica). Energy Conversión and Management.

Colowick, S. \&Kaplan, N. (1957).Methods in enzymology III: Preparation and assay of substrates. Nueva York: AcademicPress.

Córdoba, P.M (2011). Determinación del efecto de la concentración de la base $\mathrm{NaOH}$, de la celulosa y celobiasa en la hidrólisis para la producción de etanol a partir del rastrojo de la piña. Proyecto de Graduación para optar por el grado de Licenciatura en Ingeniería Química, Universidad de Costa Rica, San José, Costa Rica.
Chu, B., \& Lee, H. (2007).Genetic improvement of Saccharomyces cerevisiae for xylose fermentation.Biotechnology Advances, 25, 425-441.

Foyle, T., Jennings, L., \& Mulcahy, P. (2007). Compositional analysis of lignocellulosic materials: Evaluation of methods used for sugar analysis of waste paper and straw. Bioresource Technology, 98, 3026-3036.

Gírio, F., Fonseca, C., Carvalheiro, F., Duarte, L., Marques, S., \& Bogel-Lukasik, R. (2010). Hemicelluloses for fuel ethanol: A review. Bioresource Technology, 101, 4775-4800.

Hendricks, A., \& Zeeman, G. (2009). Pretreatments to enhance the digestibility of lignocellulosic biomass. Bioresource Technology, 100, 10-18.

Howard, R.L, Abotsi. E, Jansen Van Resbug, E., Howard, S. (2003). Lignocellulose Biotechnology: Issues of conversión and enzyme production. African Journal of Biotechnology, 2, 12, 602-619.

Kumar, P., Barret, D. M., Delwiche, M. J., \& Stroeve, P. (2009). Methods for Pretreatment of Lignocellulosic Biomass for Efficient Hydrolysis and Biofuel Production. Ind. Eng. Chem. Res., 48, 3713-3729.

MINAET, MAG, \& PROAGROIN. (2009). Evaluación del impacto ambiental generado por la eliminación del rastrojo de piña a través de su incorporación al suelo. San José.

Mosier, N., Wyman, C., Dale, B., Elander, R., Lee, Y., Holtzapple, M., y otros. (2005). Features of promising technologies for pretreatment. Bioresource Technology, 96, 673-686.

Nlewem, K. C., \& Thrash, M. E. (2010). Comparison of different pretratment methods based on residual lignin effect on the enzymatic hydrolysis of switchgrass. Bioresuorce Technology, 101, 5426-5430.

Ovando, S., \& Waliszewski, K. (2005). Preparativos de celulasas comerciales y aplicaciones en procesos extractivos. (U. J. Tabasco, Ed.) Universidad y Ciencia, 21(042), 113-122.

Quesada, K., Alvarado, P., Sibaja, R., \& Vega, J. (Junio de 2005). Utilización de las fibras del rastrojo de piña (Ananas comusus, variedad champaka) como material de refuerzo en 
resinas de poliéster. Revista Iberoamericana de Polímeros, 6(2), 158-179.

REN21. (2010). Renewables Global Status Report.

Rodríguez, A.L.(2012). Efecto del tamaño de partícula y concentración de las enzimas celulasa y celobiosa, en la hidrólisis del rastrojo de la piña para la producción de etanol. (Tesis de Licenciatura en Ingeniería Química). Universidad de Costa Rica, San José.

Southate,D.A.(1976). Determination of food carbohydrates. London: Aplied Science Publisher.

Sun, Y., Cheng, J. (2002). Hydrolysis of lignocellulosic materials for ethanol production: a review. Bioresource Technology, 83, 1-11.

Tico Lab S.A. (2002). Determinación de Glucosa: Método de Trinder. San José: Tico Lab S.A.

Torres, J.F.(2009). Evaluación del rendimiento de la hidrólisis enzimática de la celulosa a glucosa, con pretratamiento alcalino. (Tesis de Licenciatura en Ingeniería Química). Universidad de Costa Rica, San José.

Tropper, N. (2010). Evaluación del efecto de la concentración de hidróxido de sodio, relación de enzimas, tiempo de hidrólisis y la concentración de un sustrato de bagazo de caña en la hidrólisis y fermentación de un sistema integrado. (Tesis de Licenciatura en Ingeniería Química). Universidad de Costa Rica, San José.
Villegas, M. (2010). Evaluación del efecto del tamaño de partícula, concentración de enzimas y tiempo en la hidrólisis y fermentación integradas para la producción de etanol. Tesis de Licenciatura en Ingeniería Química). Universidad de Costa Rica, San José.Yu, E., Vlasenko, H., Ding, H., Labavitch, J. \& Shoemaker, S. (1997). Enzymatic hydrolysis of pretreated rice straw. Bioresource Technology, 59, 109-119.

\section{AGRADECIMIENTO}

A la Lic.Isabel Carpio M., Investigadora del Laboratorio de Productos Forestales del Instituto de Investigaciones de Ingeniería por su asesoría en el área biológica y apoyo para el tratamiento y toma de las micrografías del material lignocelulósico.

\section{SOBRE LOS AUTORES}

Maureen Córdoba Pérez. Licenciada en Ingeniería Química de la Universidad de Costa Rica. Docente de la Escuela de Ingeniería Quimica, Universidad de Costa Rica.

Correo electrónico: maureen.cordobaperez@ucr.ac.cr

Manuel Molina Córdoba. Licenciado en Ingeniería Química. Docente de la Escuela de Ingeniería Quimica, Universidad de Costa Rica. Correo electrónico: manuel.molina@ucr.ac.cr 
